\title{
Tranche Distributed Repository
}

National Cancer Institute

\section{Source}

National Cancer Institute. Tranche Distributed Repository. NCI Thesaurus. Code C81327.

A distributed repository architecture for storing and sharing large amounts of immutable data. 